\title{
Razumikhin-Type Theorems on p-th Moment Stability for Stochastic Switching Nonlinear Systems with Delay
}

\author{
Haibo Gu, Caixia Gao \\ School of Mathematical Sciences, Inner Mongolia University, Hohhot, China \\ Email: guhaibo1990@sina.com,gaocx0471@163.com
}

Received 24 March 2016; accepted 12 July 2016; published 15 July 2016

\begin{abstract}
This paper mainly tends to utilize Razumikhin-type theorems to investigate p-th moment stability for a class of stochastic switching nonlinear systems with delay. Based on the Lyapunov-Razumikhin methods, some sufficient conditions are derived to check the stability of stochastic switching nonlinear systems with delay. One numerical example is provided to demonstrate the effectiveness of the results.
\end{abstract}

\section{Keywords}

\section{Stochastic Switching Delay System, p-th Moment Stability, Lyapunov-Razumikhin Approach}

\section{Introduction}

Stochastic switching system is an indispensable class of hybrid dynamical systems, which is composed of a family of stochastic subsystems and a rule that orchestrates the switching among them. Yet, there inevitably exists delay phenomenon in the practical systems like physics, biology and economic [1] [2]. So it is important for us to study stochastic switching systems with delay. Over the previous few decades, stochastic switching delay systems have received much attention due to their potential applications in many fields, such as the control of mechanical systems, automotive industry, chemical and electrical engineering [3] [4].

It is well-known that stability is the major issue of control theory. Lyapunov-Razumikhin technique has been a powerful and effective method for investigating stability. Razumikhin developed this technique to study the stability of deterministic systems with delay in [5] [6], then, Mao extended this technique to stochastic functional differential equations [7] and neutral stochastic functional differential equations [8] to investigate p-th moment exponential stability of this systems. Later, this technique was appropriately developed and extended to some other stochastic systems, such as hybrid stochastic delay interval systems [9] and impulsive stochastic delay differential systems [10]. Recently, some researchers have introduced $\psi$-type function and extended the stability results to $\psi^{\gamma}$ stability, including the exponentialstability as a special case in [11] [12]. In [13], the researchers utilize multiple Lyapunov functions investigate the stability of stochastic switching nonlinear systems.

To the best of our knowledge, there are no results based on the Razumikhin approach referring to the $\psi^{\gamma}$ 
stability of stochastic switching nonlinear systems with delay. The main aim of this paper is to attempt to investigate p-th moment $\psi^{\gamma}$ stability of stochastic switching delay nonlinear systems. By the aid of Lyapunov-Razumikhin approach, we obtain the p-th moment $\psi^{\gamma}$ stability of stochastic switching systems with delay in Section 3. An example is presented to illustrate the main results in Section 4. Finally, the conclusions are given in Section 5 .

\section{Preliminaries}

Consider a family of stochastic switching delay nonlinear systems described by

$$
\left\{\begin{array}{l}
d x(t)=f_{\sigma(t)}\left(t, x_{t}\right) d t+g_{\sigma(t)}\left(t, x_{t}\right) d w(t) \\
x_{0}=\xi
\end{array}\right.
$$

where $\sigma(t):\left[t_{0}, \infty\right) \rightarrow N$ is the switching signal, let $\left\{t_{1}<t_{2}<\cdots t_{k}<\cdots\right\}$ be a switching sequence and the $i_{k}$ th subsystem is active at time interval $\left[t_{k}, t_{k+1}\right)$, where $t_{k}$ is the switching instant, $i_{k} \in N, k=0,1,2, \cdots$. System (1) is consisted with many stochastic subsystems $d x(t)=f_{i}\left(t, x_{t}\right) d t+g_{i}\left(t, x_{t}\right) d w(t)$ which are driven by switching signal $\sigma(t) . \xi \in C_{F_{0}}^{b}\left([-\tau, 0] ; R^{n}\right), \quad x_{t}=\{x(t+\theta):-\tau \leq \theta \leq 0\} \in C\left([-\tau, 0] ; R^{n}\right)$ and $\tau$ is finite, $w(t)$ is an m-dimensional independent standard Wiener process, and the underlying complete probability space is taken to be the quartet $\left(\Omega, F,\left\{F_{t}\right\}, P\right)$ with a filtration $F_{t}$ satisfied the usual conditions (i.e. it is increasing and right continuous while $F_{0}$ contains all P-null sets), functions $f: R_{+} \times C\left([-\tau, 0] ; R^{n}\right) \rightarrow R^{n}$,

$\mathrm{g}: R_{+} \times C\left([-\tau, 0] ; R^{n}\right) \rightarrow R^{n \times m}$ are both measurable and let $f(t, 0)=0, g(t, 0)=0, t \in\left[t_{0}, \infty\right]$.

Definition 1. $\psi(t): \psi(t) \in C^{1}\left([-\tau,+\infty) ; R_{+}\right)$is said to be $\psi$-type function, if it satisfies the following conditions:

1) It is continuous, monotone decreasing and differentiable;

2) $\psi(0)=1$ and $\psi(t) \rightarrow 0$, as $t \rightarrow \infty$;

3) $\psi_{1}(t)=\psi^{\prime}(t) / \psi(t)<0$;

4) for any $t, s \geq 0, \psi(t+s) \geq \psi(t) \psi(s)$.

Definition 2. For $p>0$, stochastic switching delay nonlinear systems (1) is said to be p-th moment $\psi^{\gamma}$ stable, if there exist positive constants $\beta, \gamma$ and function $\psi(\cdot)$, such that

$$
\limsup _{t \rightarrow \infty} \frac{\ln E|x(t, \xi)|^{p}}{|\ln \psi(t)|} \leq-\gamma .
$$

when $p=2$, we say that it is $\psi^{\gamma}$ stable in mean square, when $\psi(t)=e^{-t}$, we say that it is p-th moment exponential stable, when $\psi(t)=(1+t)^{-1}$, we say that it is p-th moment polynomial stable.

Before giving the main results, let us introduce $I t o \hat{~ f o r m u l a . ~ F o r ~ s y s t e m ~(1), ~ g i v e ~ a n y ~ f u n c t i o n ~}$ $V(t, x) \in C^{1,2}\left([-\tau,+\infty) \times R^{n} ; R_{+}\right)$and define an operator $L V(t, \varphi): R_{+} \times C\left([-\tau, 0] ; R^{n}\right) \rightarrow R$ described by

$$
L V(t, \varphi)=V_{t}(t, \varphi(0))+V_{x}(t, \varphi(0)) f(t, \varphi)+\frac{1}{2} \operatorname{tr}\left[g^{T}(t, \varphi) V_{x x}(t, \varphi(0)) g(t, \varphi)\right]
$$

where

$$
V_{t}(t, x)=\frac{\partial V(t, x)}{\partial t}, V_{x}(t, x)=\left(\frac{\partial V(t, x)}{\partial x_{1}}, \cdots, \frac{\partial V(t, x)}{\partial x_{n}}\right), V_{x x}(t, x)=\left(\frac{\partial^{2} V(t, x)}{\partial x_{i} \partial x_{j}}\right)_{n \times n}
$$

\section{Main Results}

In this section, we shall establish Razumikhin-type theorems on the p-th moment $\psi^{\gamma}$ stable for stochastic delay nonlinear systems by using Razumikhin technique and Lyapunov functions. Before giving the efficient theorem, let us give some assumptions to the switching signal $\sigma(t)$.

Assumption 1. Switching signal $\sigma(t)$ is right continuous and state-dependent.

Assumption 2. At each switching instant $t_{k},(k=1,2, \cdots \cdots)$, the state trajectory is not jumped.

Then, let us turn our attention to system (1) and give a sufficient result.

Theorem 1. For stochastic switching delay nonlinear systems (1), if there exist a group of Lyapunov func- 
tions $V_{i}(t, x) \in C^{1,2}\left([-\tau, \infty) \times R^{n} ; R_{+}\right)$and positive constants $p, b, b_{i}, c, c_{i}, \mu, q>1$, such that

$$
\begin{gathered}
b_{i}|x|^{p} \leq V_{i}(t, x) \leq c_{i}|x|^{p} \\
E L V_{i}(t, \varphi) \leq \mu \psi_{1}(t) E V_{i}(t, \varphi(0))
\end{gathered}
$$

for all $t \geq 0, \theta \in[-\tau, 0]$ and those $\varphi \in L_{F_{t}}^{p}\left([-\tau, 0] ; R^{n}\right)$ satisfying

$$
E V_{i}(t+\theta, \varphi(t+\theta)) \leq q E V_{i}(t, \varphi(0))
$$

where $q \geq \psi^{-\mu}(-\theta)$.

and at each switching instant $t_{k},(k=1,2, \cdots)$,

$$
E\left[V_{\sigma\left(t_{k+1}\right)}\left(t_{k+1}, x\left(t_{k+1}\right)\right)\right] \leq d_{k} E\left[V_{\sigma\left(t_{k}\right)}\left(t_{k}, x\left(t_{k}\right)\right)\right]
$$

where $0 \leq d_{k} \leq \psi^{\gamma}\left(t_{k+1}-t_{k}\right)$.

Then, for any initial $\xi \in C_{F_{0}}^{b}\left([-\tau, 0] ; R^{n}\right)$, there exists a solution $x(t)=x(t, \xi)$ on $\left[t_{0}, \infty\right)$ to stochastic switching delay nonlinear system (1). Moreover, the system (1) is p-th moment $\psi^{\gamma}$ stable and

$$
E|x(t, \xi)|^{p} \leq \frac{c}{b}\|\xi\|^{p} \psi^{\gamma}(t), \quad t \geq 0 .
$$

Proof. Fix the initial data $\xi \in C_{F_{0}}^{b}\left([-\tau, 0] ; R^{n}\right)$ arbitrarily and write $x(t, \xi)=x(t)$ simply. When $\mu$ is replaced by $\gamma$, if we can prove (7) for all $\gamma \in(0, \mu)$, then the desired result is obtained.

Given switching signal $\sigma(t)$ and instant $t$ for arbitrary, assume that $t_{k}$ is the last switching instant before $t$, i.e. there is no switching occur on the interval $\left[t_{k}, t\right)$.

Let $\gamma \in(0, \mu)$ be arbitrary, if we can prove

$$
b E|x(t)|^{p} \leq c\|\xi\|^{p} \psi^{\gamma}(t)
$$

we will complete this proof. By condition (6), this result follows from

$$
E V_{\sigma(t)}(t, x) \leq c_{\sigma(t)}\|\xi\|^{p} \psi^{\gamma}(t)
$$

Let $\frac{c_{i}}{b_{i}}\|\xi\|^{p}=\beta_{i}$, we have

$$
h_{\sigma(t)}(t)=\psi^{-\gamma}(t) E V_{\sigma(t)}(t, x) \leq c_{\sigma(t)}\|\xi\|^{p}=b_{\sigma(t)} \beta_{\sigma(t)}
$$

By the continuity of $h_{\sigma(t)}(t)$, it is obvious that

$$
h_{\sigma(t)}(0)=\psi^{-\gamma}(0) E V_{\sigma(t)}(0, x(0)) \leq b_{\sigma(t)} \beta_{\sigma(t)} .
$$

We claim that (8) holds for all $t \geq 0$.

In order to do so, we first prove that

$$
E V_{\sigma\left(t_{1}\right)}(t, x) \leq c_{1}\|\xi\|^{p} \psi^{\gamma}\left(t_{1}\right), \quad t \in[0, t)
$$

That is

$$
h_{\sigma\left(t_{1}\right)}(t)=\psi^{-\gamma}(t) E V_{\sigma\left(t_{1}\right)}(t, x) \leq b_{1} \beta_{1}, \quad t \in\left[0, t_{1}\right)
$$

This can be verified by a contradiction, suppose that inequality (9) is not right, then by the continuity of $h_{\sigma\left(t_{1}\right)}(t)$, there exists a smallest $t^{*} \geq 0$ such that for $\forall t \in\left[0, t^{*}\right], h_{\sigma\left(t_{1}\right)}(t) \leq c_{1} \beta_{1}$ and $h_{\sigma\left(t_{1}\right)}\left(t^{*}\right)=c_{1} \beta_{1}$ as well as $h_{\sigma\left(t_{1}\right)}\left(t^{*}+\delta\right)>h_{\sigma\left(t_{1}\right)}\left(t^{*}\right)$ for all sufficiently small $\delta$, then for $\forall \theta \in[-\tau, 0]$, if $t^{*}+\theta<0$ and $t^{*}+\theta \in[-\tau, 0]$, by condition (3), we have 


$$
\begin{aligned}
E V_{\sigma\left(t_{1}\right)}\left(t^{*}+\theta, x\left(t^{*}+\theta\right)\right) & \leq c_{1}\left\|\xi\left(t^{*}+\theta\right)\right\|^{p} \leq c_{1}\|\xi\|^{p}=b_{1} \beta_{1} \\
& =\psi^{-\gamma}\left(t^{*}\right) E V_{\sigma\left(t_{1}\right)}\left(t^{*}, x\left(t^{*}\right)\right) \leq \psi^{-\gamma}(-\theta) E V_{\sigma\left(t_{1}\right)}\left(t^{*}, x\left(t^{*}\right)\right) \\
& \leq \psi^{-\mu}(-\theta) E V_{\sigma\left(t_{1}\right)}\left(t^{*}, x\left(t^{*}\right)\right) \leq q E V_{\sigma\left(t_{1}\right)}\left(t^{*}, x\left(t^{*}\right)\right)
\end{aligned}
$$

if $t^{*}+\theta \geq 0$, then $h_{\sigma\left(t_{1}\right)}\left(t^{*}+\theta\right) \leq b_{1} \beta_{1}$ since $t^{*}+\theta \in\left[0, t^{*}\right]$, we, therefore, obtain

$$
\begin{aligned}
& E V_{\sigma\left(t_{1}\right)}\left(t^{*}+\theta, x\left(t^{*}+\theta\right)\right) \leq \psi^{\gamma}\left(t^{*}+\theta\right) h_{\sigma\left(t_{1}\right)}\left(t^{*}+\theta\right) \leq \psi^{\gamma}\left(t^{*}+\theta\right) b_{1} \beta_{1} \\
& =\psi^{\gamma}\left(t^{*}+\theta\right) \psi^{-\gamma}\left(t^{*}\right) E V_{\sigma\left(t_{1}\right)}\left(t^{*}, x\left(t^{*}\right)\right) \leq \psi^{-\gamma}(-\theta) E V_{\sigma\left(t_{1}\right)}\left(t^{*}, x\left(t^{*}\right)\right) \\
& \leq \psi^{-\mu}(-\theta) E V_{\sigma\left(t_{1}\right)}\left(t^{*}, x\left(t^{*}\right)\right) \leq q E V_{\sigma\left(t_{1}\right)}\left(t^{*}, x\left(t^{*}\right)\right)
\end{aligned}
$$

Therefore, for $\forall \theta \in[-\tau, 0]$, we have

$$
E V_{\sigma\left(t_{1}\right)}\left(t^{*}+\theta, x\left(t^{*}+\theta\right)\right) \leq q E V_{\sigma\left(t_{1}\right)}\left(t^{*}, x\left(t^{*}\right)\right)
$$

By condition (4), we can obtain

$$
E L V_{\sigma\left(t_{1}\right)}\left(t^{*}, x_{t^{*}}\right) \leq \mu \psi_{1}\left(t^{*}\right) E V_{\sigma\left(t_{1}\right)}\left(t^{*}, x\left(t^{*}\right)\right)
$$

By the continuity of $V_{\sigma\left(t_{1}\right)}(t, x)$, for all sufficiently small $\delta$, when $t \in\left[t^{*}, t^{*}+\delta\right]$, we have

$$
E L V_{\sigma\left(t_{1}\right)}(t, \varphi) \leq \mu \psi_{1}(t) E V_{\sigma\left(t_{1}\right)}(t, x(t))
$$

By the Itô formula and continuity of $h_{\sigma\left(t_{1}\right)}(t)$, for all sufficiently small $\delta$, we can obtain

$$
h_{\sigma\left(t_{1}\right)}\left(t^{*}+\delta\right)-h_{\sigma\left(t_{1}\right)}\left(t^{*}\right)=\int_{t^{*}}^{t^{*}+\delta} \psi^{-\gamma}(t)\left(E L V_{\sigma\left(t_{1}\right)}\left(t, x_{t}\right)-\gamma \psi_{1}(t) E V_{\sigma\left(t_{1}\right)}(t, x(t))\right) d t
$$

By condition (4)

$$
h_{\sigma\left(t_{1}\right)}\left(t^{*}+\delta\right)-h_{\sigma\left(t_{1}\right)}\left(t^{*}\right) \leq 0
$$

which is a contradiction. Hence, inequality (9) holds for all $t \geq 0$, and inequality (8) is right for $k=1$.

Now, let $c=\max \left\{c_{i}\right\}$. We assume that inequality (8) holds for $k=1,2, \cdots, m,(m \in N, m \geq 1)$, i.e.

$$
E V_{\sigma\left(t_{k}\right)}(t, x) \leq c\|\xi\|^{p} \psi^{\gamma}\left(t_{k}\right), \quad t \in\left[t_{k-1}, t_{k}\right), k=1,2, \cdots, m
$$

That is

$$
h_{\sigma\left(t_{k}\right)}(t)=\psi^{-\gamma}(t) E V_{\sigma\left(t_{k}\right)}(t, x) \leq b_{\sigma\left(t_{k}\right)} \beta_{\sigma\left(t_{k}\right)}, \quad t \in\left[t_{k-1}, t_{k}\right), k=1,2, \cdots, m
$$

We will prove that

$$
h_{\sigma\left(t_{m+1}\right)}(t)=\psi^{-\gamma}\left(t_{t_{m+1}}\right) E V_{\sigma\left(t_{m+1}\right)}(t, x) \leq b_{\sigma\left(t_{m+1}\right)} \beta_{\sigma\left(t_{m+1}\right)}, \quad t \in\left[t_{m}, t_{m+1}\right)
$$

Suppose that inequality (14) is not right,

By condition (6) and inequality (12), we have

$$
E V_{\sigma\left(t_{m+1}\right)}\left(t_{m+1}, x\left(t_{m+1}\right)\right) \leq d_{m} E V_{\sigma\left(t_{m}\right)}\left(t_{m}, x\left(t_{m}\right)\right) \leq d_{m} c\|\xi\|^{p} \psi^{\gamma}\left(t_{m}\right) \leq \psi^{\gamma}\left(t_{m}\right) c\|\xi\|^{p} \psi^{\gamma}\left(t_{m+1}-t_{m}\right) \leq c\|\xi\|^{p} \psi^{\gamma}\left(t_{m+1}\right)
$$

That is

$$
h_{\sigma\left(t_{m+1}\right)}(t)=\psi^{-\gamma}\left(t_{m+1}\right) E V_{\sigma\left(t_{m+1}\right)}(t, x) \leq c_{\sigma\left(t_{m+1}\right)}\|\xi\|^{p}=b_{\sigma\left(t_{m+1}\right)} \beta_{\sigma\left(t_{m+1}\right)}
$$

Then by the continuity of $h_{\sigma\left(t_{m+1}\right)}(t)$, there exists a smallest $t^{*} \in\left(t_{m}, t_{m+1}\right)$ such that for $\forall t \in\left[t_{m}, t^{*}\right]$, $h_{\sigma\left(t_{m+1}\right)}(t) \leq b_{\sigma\left(t_{m+1}\right)} \beta_{\sigma\left(t_{m+1}\right)}$ and $h_{\sigma\left(t_{m+1}\right)}\left(t^{*}\right)=b_{\sigma\left(t_{m+1}\right)} \beta_{\sigma\left(t_{m+1}\right)}$ as well as $h_{\sigma\left(t_{m+1}\right)}\left(t^{*}+\delta\right)>h_{\sigma\left(t_{m+1}\right)}\left(t^{*}\right)$ for all sufficiently small $\delta$, then for $\forall \theta \in[-\tau, 0]$, if $t^{*}+\theta<t_{m}$, then from (15), we have 


$$
\begin{aligned}
& E V_{\sigma\left(t_{m+1}\right)}\left(t^{*}+\theta, x\left(t^{*}+\theta\right)\right) \leq c_{\sigma\left(t_{m+1}\right)}\|\xi\|^{p} \psi^{\gamma}\left(t^{*}+\theta\right)=b_{\sigma\left(t_{m+1}\right)} \beta_{\sigma\left(t_{m+1}\right)} \psi^{\gamma}\left(t^{*}+\theta\right) \\
= & h_{\sigma\left(t_{m+1}\right)}\left(t^{*}\right) \psi^{\gamma}\left(t^{*}+\theta\right)=\psi^{-\gamma}\left(t^{*}\right) E V_{\sigma\left(t_{m+1}\right)}\left(t^{*}, x\left(t^{*}\right)\right) \psi^{\gamma}\left(t^{*}+\theta\right) \leq \psi^{-\gamma}(-\theta) E V_{\sigma\left(t_{m+1}\right)}\left(t^{*}, x\left(t^{*}\right)\right) \\
\leq & \psi^{-\mu}(-\theta) E V_{\sigma\left(t_{m+1}\right)}\left(t^{*}, x\left(t^{*}\right)\right) \leq q E V_{\sigma\left(t_{m+1}\right)}\left(t^{*}, x\left(t^{*}\right)\right)
\end{aligned}
$$

if $t^{*}+\theta \geq t_{m}$, then $h_{\sigma\left(t_{m+1}\right)}\left(t^{*}+\theta\right) \leq b_{\sigma\left(t_{m+1}\right)} \beta_{\sigma\left(t_{m+1}\right)}$ since $t^{*}+\theta \in\left[t_{m}, t^{*}\right]$, we, therefore, obtain

$$
\begin{aligned}
& E V_{\sigma\left(t_{m+1}\right)}\left(t^{*}+\theta, x\left(t^{*}+\theta\right)\right) \leq \psi^{\gamma}\left(t^{*}+\theta\right) h_{\sigma\left(t_{m+1}\right)}\left(t^{*}+\theta\right) \leq \psi^{\gamma}\left(t^{*}+\theta\right) b_{\sigma\left(t_{m+1}\right)} \beta_{\sigma\left(t_{m+1}\right)} \\
= & \psi^{\gamma}\left(t^{*}+\theta\right) h_{\sigma\left(t_{m+1}\right)}\left(t^{*}\right) \leq \psi^{\gamma}\left(t^{*}+\theta\right) \psi^{-\gamma}\left(t^{*}\right) E V_{\sigma\left(t_{m+1}\right)}\left(t^{*}, x\left(t^{*}\right)\right) \leq \psi^{-\gamma}(-\theta) E V_{\sigma\left(t_{m+1}\right)}\left(t^{*}, x\left(t^{*}\right)\right) \\
\leq & \psi^{-\mu}(-\theta) E V_{\sigma\left(t_{m+1}\right)}\left(t^{*}, x\left(t^{*}\right)\right) \leq q E V_{\sigma\left(t_{m+1}\right)}\left(t^{*}, x\left(t^{*}\right)\right)
\end{aligned}
$$

Therefore, for $\forall \theta \in[-\tau, 0]$, we have

$$
E V_{\sigma\left(t_{m+1}\right)}\left(t^{*}+\theta, x\left(t^{*}+\theta\right)\right) \leq q E V_{\sigma\left(t_{m+1}\right)}\left(t^{*}, x\left(t^{*}\right)\right)
$$

By condition (4), we can obtain

$$
E L V_{\sigma\left(t_{m+1}\right)}\left(t^{*}, x_{t^{*}}\right) \leq \mu \psi_{1}\left(t^{*}\right) E V_{\sigma\left(t_{m+1}\right)}\left(t^{*}, x\left(t^{*}\right)\right)
$$

By the continuity of $V_{\sigma\left(t_{m+1}\right)}(t, x)$, for all sufficiently small $\delta$, when $t \in\left[t^{*}, t^{*}+\delta\right]$, we have

$$
E L V_{\sigma\left(t_{m+1}\right)}(t, \varphi) \leq \mu \psi_{1}(t) E V_{\sigma\left(t_{m+1}\right)}(t, x(t))
$$

By the Itô formula and continuity of $h_{\sigma\left(t_{m+1}\right)}(t)$, for all sufficiently small $\delta$, we can obtain

$$
h_{\sigma\left(t_{m+1}\right)}\left(t^{*}+\delta\right)-h_{\sigma\left(t_{m+1}\right)}\left(t^{*}\right)=\int_{t^{*}}^{t^{*}+\delta} \psi^{-\gamma}(t)\left(E L V_{\sigma\left(t_{m+1}\right)}\left(t, x_{t}\right)-\gamma \psi_{1}(t) E V_{\sigma\left(t_{m+1}\right)}(t, x(t))\right) d t
$$

By condition (4)

$$
h_{\sigma\left(t_{m+1}\right)}\left(t^{*}+\delta\right)-h_{\sigma\left(t_{m+1}\right)}\left(t^{*}\right) \leq 0
$$

which is a contradiction. Hence, inequality (14) holds for all $t \geq 0$.

Therefore, by mathematical induction we obtain (8) holds for all $k \in N$.

Then, $\forall \theta \in[-\tau, 0]$, we have

$$
E|x(t, \xi)|^{p} \leq \frac{C_{\sigma\left(t_{k}\right)}}{b_{\sigma\left(t_{k}\right)}}\|\xi\|^{p} \psi^{\gamma}(t)
$$

That is

$$
E|x(t, \xi)|^{p} \leq \frac{c}{b}\|\xi\|^{p} \psi^{\gamma}(t), \quad t \geq 0 .
$$

Thus, the system (1) is p-th moment $\psi^{\gamma}$ stable.

\section{Example}

In this section, a numerical example is given to illustrate the effectiveness of the main results established in Section 3 as follows.

Consider a family of stochastic switching delay nonlinear systems

$$
d x(t)=f_{\sigma(t)}\left(t, x_{t}\right) d t+g_{\sigma(t)}\left(t, x_{t}\right) d w(t), \quad t \geq 0
$$

where $\sigma(t):\left[t_{0}, \infty\right) \rightarrow\{1,2\}$ is switching signal. Let $\left\{t_{1}<t_{2}<\cdots<t_{k}<\cdots\right\}$ be a switching sequence and the $i_{k}$ th subsystem is active at time interval $\left[t_{k}, t_{k+1}\right)$, where $t_{k}$ is switching instant, $k=0,1,2, \cdots$ and $i_{k} \in\{1,2\}$. 
We choose $d_{k}=0.15, t_{k+1}-t_{k}=0.5 s, \tau=0.1, q=1.6, \psi(t)=\frac{e^{-t}}{1+t}, t \geq 0$, then, $\psi(0)=1, \psi(\infty)=0$, $\psi^{\prime}(t)=-\frac{2+t}{(1+t)^{2}} e^{-t}, \quad \psi_{1}(t)=-\left(1+\frac{1}{1+t}\right), \quad-2 \leq \psi_{1}(t) \leq-1$.

When $\sigma(t)=1$, we choose $f_{1}(t, x)=-3 x(t)-\operatorname{sgn} x(t)+0.625 x(t-0.1)$ and $g_{1}(t, x)=\sqrt{2 x(t)}$ for the first subsystem; when $\sigma(t)=2$, we choose $f_{2}(t, x)=-7 x+0.625 x(t-0.1)$ and $g_{2}(t, x)=\sqrt{5} x(t-0.1)$ for the second subsystem.

For the first subsystem, we choose $V_{1}(t, x)=8 x^{2}$, then $x^{2} \leq V_{1}(t, x) \leq 8 x^{2}$. If $x \neq 0$, we have

$$
\begin{aligned}
E L V_{1}\left(t, x_{t}\right) & =E\left[16 x(-3 x(t)-\operatorname{sgn} x(t)+0.625 x(t-0.1))+\frac{1}{2} \cdot 16 \cdot(\sqrt{2 x(t)})^{2}\right] \\
& \leq-48 E|x(t)|^{2}+10 q E|x(t)|^{2}=-4 E V_{1}(t, x) \leq 2 \psi_{1}(t) E V_{1}(t, x)
\end{aligned}
$$

If $x=0$, then $V_{1}(t, x)=0, E L V_{1}(t, x)=0$.

For the second subsystem, we choose $V_{2}(t, x)=x^{2}$, then $\frac{1}{2} x^{2} \leq V_{2}(t, x) \leq x^{2}$. If $x \neq 0$, we have

$$
\begin{aligned}
E L V_{2}\left(t, x_{t}\right) & =E\left[2 x \cdot(-7 x+0.625 x(t-0.1))+(\sqrt{5} x(t-0.1))^{2}\right] \\
& \leq-14 E|x(t)|^{2}+6.25 q E|x(t)|^{2}=-4 E V_{2}(t, x) \leq 2 \psi_{1}(t) E V_{2}(t, x)
\end{aligned}
$$

If $x=0$, then $V_{2}(t, x)=0, E L V_{2}(t, x)=0$.

By Theorem 1, we can choose $p=2, \gamma=2$, then $E L V_{i}\left(t, x_{t}\right) \leq \gamma \psi_{1}(t) E V_{i}(t, x), i=1,2$, which means that the conditions of Theorem 1 are satisfied. So the stochastic switching delay nonlinear systems are p-th moment $\psi^{\gamma}$ stability. The switching signal and the state trajectory are presented in Figure $\mathbf{1}$ and Figure 2, respectively.

Remark. In the example, a stochastic switching delay nonlinear system is constructed to show the efficiency of the results. Figure 1 describes switching signal changes over the time. Figure $\mathbf{2}$ depicts state trajectory changes over the time, the blue line describes the systems with delay and the red describes the systems without delay.

\section{Conclusion}

In this paper, p-th moment $\psi^{\gamma}$ stability has been investigated for stochastic switching nonlinear systems with delay. Some sufficient conditions have been derived to check the stability criteria by using the Lyapunov-Ra-

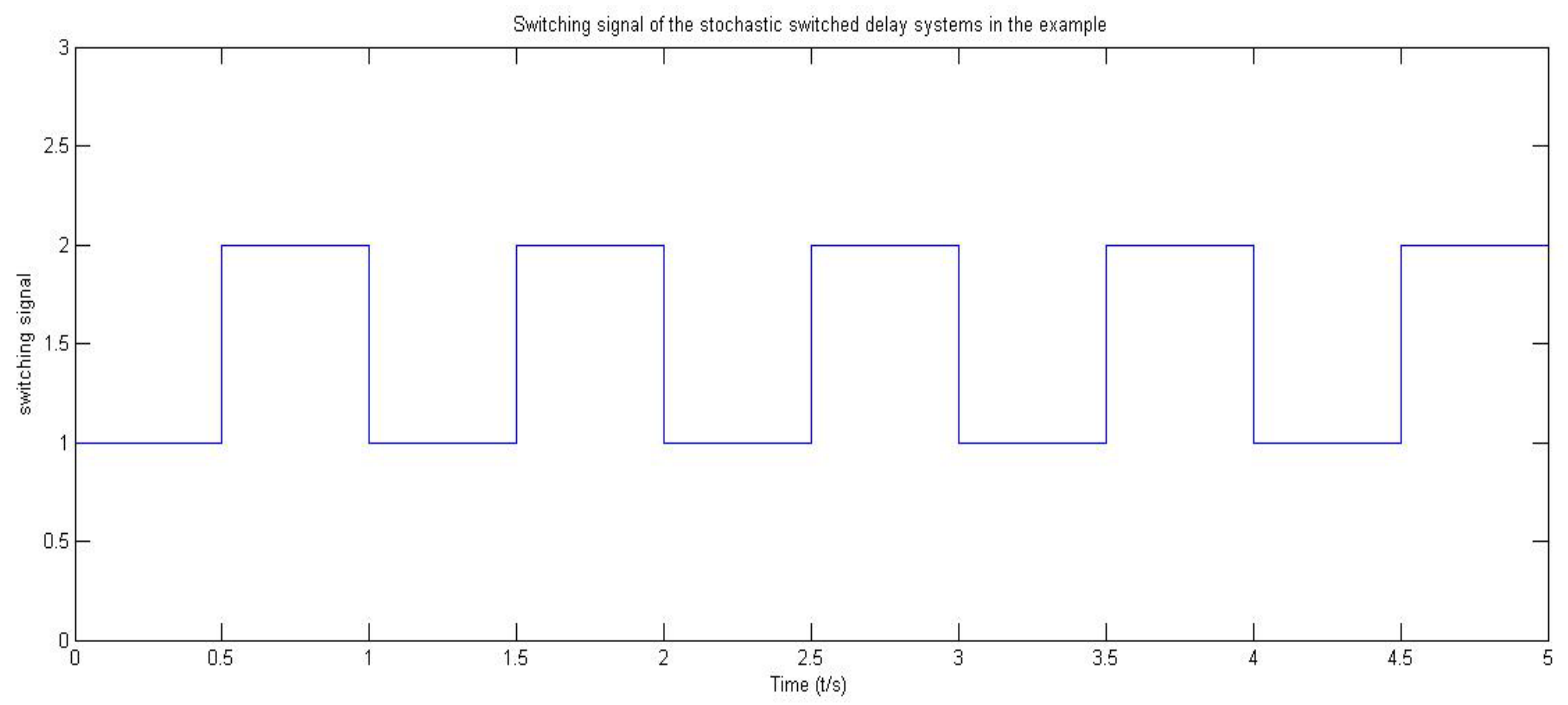

Figure 1. Switching signal of the stochastic switching systems with delay. 


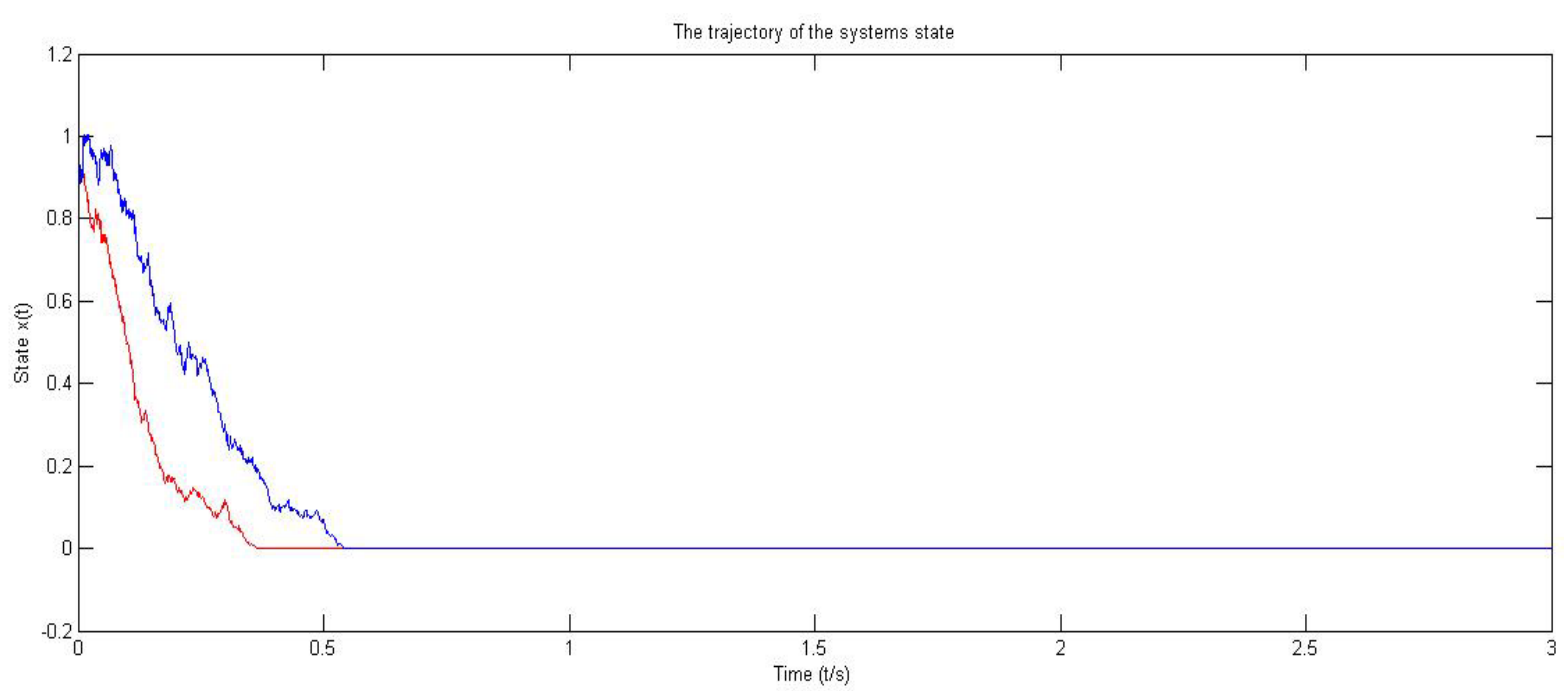

Figure 2. The trajectory of the stochastic switching delay systems’ state.

zumikhin methods. A numerical example is provided to verify the effectiveness of the main results. Our future research will focus on $\psi^{\gamma}$ stability of neutral stochastic switched nonlinear systems and $\psi^{\gamma}$ stability of impulsive stochastic switched delay nonlinear systems.

\section{Acknowledgements}

The work was supported by the National Natural Science Foundation of China under Grants 11261033 and the Postgraduate Scientific Research Innovation Foundation of Inner Mongolia under Grant 1402020201336.

\section{References}

[1] Hale, J.K. and Lunel, S.M.V. (1993) Introduction to Functional Differential Equations. Springer-Verlag Berlin. http://dx.doi.org/10.1007/978-1-4612-4342-7

[2] Feng, W., Tian, J. and Zhao, P. (2011) Stability Analysis of Switched Stochastic Systems. Automatica, 47, 148-157. http://dx.doi.org/10.1016/j.automatica.2010.10.023

[3] Liu, J., Liu, X. and Xie, W. (2009) Exponential Stability of Switched Stochastic Delay Systems with Non-Linear Uncertainties. International Journal of Systems Science, 40, 637-648. http://dx.doi.org/10.1080/00207720902755770

[4] Kolmanovskii, V.B. and Nosov, V.R. (1986) Stability of Functional Differential Equations. Academic Press, INC, New York.

[5] Razumikhin, B.S. (1956) On the Stability of Systems with Delay. Prikl. Mat. Mekh, 20, 500-512.

[6] Razumikhin, B.S. (1960) Application of Lyapunov's Method to Problems in the Stability of Systems with a Delay. Automat, i Telemekh, 21, 740-749.

[7] Mao, X. (1996) Razumikhin-Type Theorems on Exponential Stability of Stochastic Functional Differential Equations. Stochastic Processes and their Applications, 65, 233-250. http://dx.doi.org/10.1016/S0304-4149(96)00109-3

[8] Mao, X. (1997) Razumikhin-type Theorems on Exponential Stability of Neutral Stochastic Functional Differential Equations. SIAM: SIAM Journal on Mathematical Analysis, 28, 389-401. http://dx.doi.org/10.1137/S0036141095290835

[9] Mao, X., Lam, J. and Xu, S. (2006) Razumikhin Method and Exponential Stability of Hybrid Stochastic Delay Interval Systems. Journal of Mathematical Analysis and Applications, 314, 45-66. http://dx.doi.org/10.1016/j.jmaa.2005.03.056

[10] Wu, X., Zhang, W. and Tang, Y. (2013) pth Moment Stability of Impulsive Stochastic Delay Differential Systems with Markovian Switching. Communications in Nonlinear Science and Numerical Simulation, 18, 1870-1879. http://dx.doi.org/10.1016/j.cnsns.2012.12.001

[11] Hu, S., Huang, C. and Wu, F. (2008) Stochastic Differential Equations. Science Press, Beijing.

[12] Wu, F. and Hu, S. (2012) Razumikhin-Type Theorems on General Decay Stability and Robustness for Stochastic Functional Differential Equations. International Journal of Robust and Nonlinear Control, 22, 763-777. $\underline{\text { http://dx.doi.org/10.1002/rnc.1726 }}$ 
[13] Chatterjee, D. and Liberzon, D. (2006) Stability Analysis of Deterministic and Stochastic Switched Systems via a Comparison Principle and Multiple Lyapunov Functions. SIAM Journal on Control and Optimization, 45, 174-206. http://dx.doi.org/10.1137/040619429

\section{Submit or recommend next manuscript to SCIRP and we will provide best service for you:}

Accepting pre-submission inquiries through Email, Facebook, LinkedIn, Twitter, etc.

A wide selection of journals (inclusive of 9 subjects, more than 200 journals)

Providing 24-hour high-quality service

User-friendly online submission system

Fair and swift peer-review system

Efficient typesetting and proofreading procedure

Display of the result of downloads and visits, as well as the number of cited articles

Maximum dissemination of your research work

Submit your manuscript at: http://papersubmission.scirp.org/ 